\title{
Maternal anti-D prophylaxis during pregnancy does not cause neonatal haemolysis
}

\author{
A Maayan-Metzger, T Schwartz, J Sulkes, P Merlob
}

\begin{abstract}
Objective-To evaluate signs of haemolysis in babies of Rh-D negative mothers who underwent prophylaxis with anti-D immunoglobulin during pregnancy.

Design-The following were evaluated in all babies of Rh-D negative mothers born within a three month period in our department: haemoglobin level, packed cell volume, mean corpuscular volume, reticulocytes, bilirubin level, and direct Coombs' test (direct anti-globulin test). The babies were divided into two groups according to number of doses of anti-D immunoglobulin received by the mother (one or two), and then further divided by their $\mathrm{Rh}$ status (negative or positive). Findings were also compared with a control group of babies of $\mathrm{O}-\mathrm{Rh}$ positive mothers.
\end{abstract}

Results-The study group consisted of 101 babies and the control group of 37 babies. No statistically significant differences were found for any of the haematological variables between the babies of mothers who received one or two doses of anti-D immunoglobulin, or between the Rh negative babies $(n=35)$, and the controls. Although $20 \%$ of the $\mathrm{Rh}$ positive babies born to mothers receiving two doses of anti-D immunoglobulin had a positive result in the direct Coombs' test compared with only $2.4 \%$ of the babies of mothers treated with only one dose, no signs of haemolysis were documented in the babies with a positive Coombs test.

Conclusion-The prevention of $\mathrm{Rh}$ isoimmunisation with anti-D immunoglobulin (one or two doses) during pregnancy does not jeopardise the newborn. Blood group typing and direct Coombs' test should be performed in every newborn of an $\mathrm{Rh}$ negative mother to establish whether there is a necessity to administer anti-D. In the presence of a positive direct Coombs' test, the type of antibodies should be identified.

(Arch Dis Child Fetal Neonatal Ed 2001;84:F60-F62)

Keywords: Rh incompatibility; anti-D prophylaxis; haemolysis; newborn

$\mathrm{Rh}$ disease occurs in $\mathrm{Rh}-\mathrm{D}$ positive fetuses of $\mathrm{Rh}$ negative mothers who were immunised by transplacental passage of $\mathrm{Rh}-\mathrm{D}$ positive fetal red blood cells during a previous pregnancy. In such cases, maternal IgG antibodies to Rh-D cross the placenta, coating and destroying the $\mathrm{Rh}-\mathrm{D}$ positive fetal red blood cells. Until three decades ago, before the use of aggressive fetal and neonatal treatment, haemolytic disease of the newborn due to $\mathrm{Rh}$ incompatibility was a major cause of death from hydrops in $25 \%$ of affected fetuses and death from kernicterus in $25 \%$ of severely affected neonates. ${ }^{12}$ In 1968 , Bowman $^{2}$ reported that this disease was preventable by injection with $\mathrm{RhO}(\mathrm{D})$ immunoglobulin immediately after birth to Rh-D negative mothers who had given birth to an Rh-D positive infant. This treatment was later recommended at the McMaster Conference on Prevention of $\mathrm{Rh}$ Immunisation. ${ }^{3}$ A more recent statement on routine antenatal prophylaxis has been published in the United Kingdom. ${ }^{4}$ Unless the father is known to be $\mathrm{Rh}-\mathrm{D}$ negative, the mother must receive $\mathrm{RhO}(\mathrm{D})$ immunoglobulin (at a dose of 500 IU), at 28 or 34 weeks gestation, and also after abortion, amniocentesis, chorionic villi sampling, or any other invasive intrauterine procedure (cordocentesis, external version, maternal trauma, etc). According to Thornton et al, ${ }^{5}$ the effect of anti-D immunoglobulin injection persists at least into the second pregnancy. The increase in this practice has led to more pregnant women being exposed to two or more doses of anti-D immunoglobulin before delivery. Because anti-D antibodies can cross the placenta and raise fetal antibody titres, their injection increases the potential risk of haemolytic damage to $\mathrm{RhD}$ positive fetuses.

This problem has to date received little attention. The aim of this study was to evaluate markers of haemolysis in babies of $\mathrm{RhD}$ negative mothers given one or two doses of $\mathrm{RhO}(\mathrm{D})$ immunoglobulin.

\section{Materials and methods}

The study group included all babies born to $\mathrm{Rh}$ negative mothers at the Rabin Medical Center, Beilinson Campus, Petah Tiqva, Israel within a three month period (1 January to 31 March 1999). For each mother, number of previous pregnancies and births and number of doses of anti-D immunoglobulin received during the present pregnancy were recorded. For all babies, in addition to the routine blood group typing and Coombs' test, blood smear was performed at age 12-36 hours, and haemoglobin, packed cell volume, mean corpuscular volume, percentage reticulocytes, and bilirubin level were recorded, as were gestational age and birth weight. The study was approved by the ethics committee at Rabin Medical Center, and all parents gave informed consent.

The infants were grouped according to the number of maternal doses of anti-D immunoglobulin: one (routine prophylaxis at 28 
Table 1 Clinical details of the four groups

\begin{tabular}{|c|c|c|c|c|c|}
\hline & Group $1(n=41)$ & Group $2(n=25)$ & Group $3(n=35)$ & Group $4(n=37)$ & $p$ Value \\
\hline Mother & $\mathrm{Rh}-$ & $\mathrm{Rh}-$ & $\mathrm{Rh}-$ & $\mathrm{O}+$ & \\
\hline Infant & $\mathrm{Rh}+$ & $\mathrm{Rh}+$ & $\mathrm{Rh}-$ & $\mathrm{Rh}+$ or $\mathrm{Rh}-$ & \\
\hline Anti-D doses & 1 & 2 & 1 or 2 & None & \\
\hline \multicolumn{6}{|l|}{ Mother } \\
\hline Gravida & $3(2)$ & $26.5(1.5)$ & $26.5(1.5)$ & $3(2.5)$ & 0.8 \\
\hline Para & $2.5(1.7)$ & $2(1.7)$ & $2.2(1.3)$ & $2.5(2.1)$ & 0.6 \\
\hline \multicolumn{6}{|l|}{ Infant } \\
\hline Gestational age at delivery (weeks) & $39.7(1.1)$ & $38.64(1.2)$ & $39.4(1.2)$ & $39.2(1.3)$ & 0.01 \\
\hline Birth weight $(\mathrm{g})$ & $3293(416)$ & $3056(574)$ & $3381(505)$ & $3297(435)$ & 0.07 \\
\hline $\operatorname{Sex}(M / F)$ & $18 / 23$ & $7 / 18$ & $16 / 19$ & $20 / 17$ & 0.25 \\
\hline
\end{tabular}

Values are means (SD). The statistical test used was analysis of variance using the Duncan multiple comparison option.

weeks gestation) or two (standard prophylaxis plus extra dose after amniocentesis). Each of these groups was further divided by $\mathrm{Rh}$ status (positive or negative). The $\mathrm{Rh}$ negative subgroup later served as the control group for comparison of the haematological variables, as anti-D immunoglobulin cannot cause haemolysis in $\mathrm{Rh}$ negative babies. The results were also compared with a second control group of babies born to $\mathrm{O}-\mathrm{Rh}$ positive mothers who underwent blood grouping and Coombs' test as routine procedures to detect possible $\mathrm{ABO}$ incompatibility. Blood smear was performed in this group as well.

Babies born to mothers with perinatal fever, premature rupture of the membranes of more than 24 hours duration, diabetes, or any other chronic disease that could influence neonatal haematological variables were excluded, as were babies with major congenital malformations, fetal distress, or respiratory distress after birth. In addition, we excluded babies with type A or B blood born to mothers with type O blood in order to avoid double incompatibility. In the $\mathrm{O}-\mathrm{Rh}$ positive control group, we excluded babies with positive Coombs' test or with early jaundice.

STATISTICAL ANALYSIS

Results are given as mean (SD). $\chi^{2}$ test or Fisher's exact test was used as appropriate to analyse statistically significant relations between the distribution of categorical variables. Student's $t$ test was used to compare significant differences in mean continuous variables between two groups, and analysis of variance with the Duncan multiple comparison option was used to compare significant differences in mean continuous variables between three groups. $p$ Values $\leqslant 0.05$ were considered statistically significant.

Table 2 Haematological data for the four groups

\begin{tabular}{|c|c|c|c|c|c|}
\hline & $\begin{array}{l}\text { Group } 1 \\
(n=41)\end{array}$ & $\begin{array}{l}\text { Group } 2 \\
(n=25)\end{array}$ & $\begin{array}{l}\text { Group } 3 \\
(n=35)\end{array}$ & $\begin{array}{l}\text { Group } 4 \\
(n=37)\end{array}$ & $p$ Value \\
\hline Mother & Rh- & $\mathrm{Rh}-$ & $\mathrm{Rh}-$ & $\mathrm{O}+$ & \\
\hline Infant & $\mathrm{Rh}+$ & $\mathrm{Rh}+$ & $\mathrm{Rh}-$ & $\mathrm{Rh}+$ or $\mathrm{Rh}-$ & \\
\hline Anti-D doses & 1 & 2 & 1 or 2 & None & \\
\hline Haemoglobin $(\mathrm{g} / 100 \mathrm{ml})$ & $19.5(2.0)$ & $19.2(2.2)$ & $19.2(2.5)$ & $18.7(2.2)$ & 0.51 \\
\hline Packed cell volume & $60.2(6.6)$ & $58.8(7.0)$ & $59(8.3)$ & $57.6(7.0)$ & 0.47 \\
\hline $\operatorname{MCV}\left(\mu \mathrm{m}^{3}\right)$ & $107.3(4.7)$ & $109.1(4.4)$ & $105(6.0)$ & $106(5.0)$ & 0.02 \\
\hline Reticulocytes (\%) & $5.5(1.2)$ & $5.4(1.3)$ & $4.9(1.4)$ & $4.6(1.3)$ & 0.1 \\
\hline No with hyperbilirubinaemia $(>12 \mathrm{mg} / \mathrm{dl}$ ) & $2(4.8 \%)$ & $2(8 \%)$ & $2(5.7 \%)$ & Not done & 0.87 \\
\hline No with direct Coombs positive & $1(2.4 \%)$ & $5(20 \%)$ & 0 & 0 & 0.025 \\
\hline
\end{tabular}

Values are means (SD). The statistical test used was analysis of variance using the Duncan multiple comparison option. $\mathrm{MCV}$, Mean corpuscular volume.

\section{Results}

A total of 138 full term infants were included in the study, divided into four groups: group 1, 41 $\mathrm{Rh}$ positive infants born to $\mathrm{Rh}$ negative mothers who had received one dose of anti-D immunoglobulin during pregnancy; group 2, $25 \mathrm{Rh}$ positive infants born to $\mathrm{Rh}$ negative mothers who had received two doses of anti-D immunoglobulin during pregnancy; group 3, $35 \mathrm{Rh}$ negative infants born to $\mathrm{Rh}$ negative mothers who had received one or two doses of anti-D immunoglobulin during pregnancy (first control group); group 4, 37 infants born to $\mathrm{O}-\mathrm{Rh}$ positive mothers with no ABO incompatibility (second control group).

Table 1 presents the characteristics of the four groups. No statistically significant differences were found among the groups with regard to number of gestations and parity of the mothers and birth weight and sex of the newborns ( $p=0.8,0.6,0.07,0.25$ respectively). However, mean gestational age was slightly lower in group 2 than in groups 1,3 and 4 , which were similar for this variable $(p=0.01)$.

Table 2 contains the haematological findings for the four groups. There were no statistically significant differences among the groups with regard to mean haemoglobin, mean packed cell volume, or percentage reticulocytes $(p=0.51$, $0.47,0.10$, respectively). Mean corpuscular volume was higher in group 2 than in group 3 $(\mathrm{p}=0.02)$.

Six babies in groups 1,2 , and 3 (two in each group) had neonatal jaundice, with bilirubin levels higher than $12 \mathrm{mg} / \mathrm{dl}$. Three of these babies had polycythaemia (venous packed cell volume higher than $65 \%$ ).

Coombs' test, performed in the 101 babies born to $\mathrm{Rh}$ negative mothers, was positive in five of the 25 infants $(20 \%)$ whose mothers had 
received two doses of anti-D immunoglobulin (group 2) compared with only one of the 41 infants $(2 \%)$ whose mothers had received only one dose (group 1). In all infants with positive Coombs' test, antibody identification disclosed only anti-D immunoglobulin. Although mean gestational age (38.5 weeks) and birth weight (2882 g) were lower in the babies with a positive Coombs test, mean packed cell volume $(60.25 \%)$, mean haemoglobin $(19.8 \mathrm{~g} / \mathrm{dl})$, percentage reticulocytes (4.4), and presence of neonatal jaundice were similar to the findings for the babies with a negative Coombs' test.

\section{Discussion}

To the best of our knowledge, the fetal risk of haemolysis due to high maternal doses of anti-D immunoglobulin has hardly been explored. However, the current dose was extensively studied before clinical use. Hermann and Kjellman ${ }^{6}$ found no changes in cord packed cell volume or bilirubin concentration in 510 babies of mothers who had received a single dose of anti-D immunoglobulin during pregnancy. No prospective studies have evaluated haematological variables as signs of haemolysis in newborns of women treated with multiple doses.

Our study showed no significant differences, compared with controls, in haemoglobin, packed cell volume, or percentage reticulocytes in $\mathrm{Rh}$ positive babies born to $\mathrm{Rh}$ negative mothers who had received one or two doses of anti-D immunoglobulin. This was also true when the six babies of the $66 \mathrm{Rh}$ negative mothers $(9 \%)$ who had a positive direct Coombs' test were analysed separately. Furthermore, even though there were significantly more positive direct Coombs' tests among the babies of mothers who had received two doses of anti-D immunoglobulin (20\%) than among babies whose mothers had received only one dose $(2 \%)$, their haematological variables were similar. These findings indicate that the placental passage of anti-D immunoglobulin does not produce haematological changes relevant to haemolysis.

We therefore conclude that the prevention of $\mathrm{Rh}$ isoimmunisation by the injection of anti-D immunoglobulin, whether one or two doses, does not jeopardise newborns, even in the event of transplacental passage of anti-D immunoglobulin ( $9 \%$ of cases).

Our study supports the routine practice of blood group typing and direct Coombs' testing in every baby of an Rh negative mother immediately after birth to establish the necessity to administer anti-D immunoglobulin. It does not support the practice of taking a blood count in these babies as a routine procedure. In the presence of a positive direct Coombs' test, the antibodies need to be identified as Rh-D type to exclude the possibility of other minor blood group incompatibilities; of course, in this special situation, early jaundice and haematological signs of haemolysis will be present.

We thank Gloria Ginzach and Marian Propp for their editorial and secretarial assistance.

1 Bowman JM. RhD hemolytic disease of the newborn. $N$ Engl f Med 1998;339:1775-6.

2 Bowman JM. The prevention of Rh immunization. Transfus Med Rev 1988;2:129-50.

3 McMaster Conference on Prevention of $\mathrm{Rh}$ immunization. Vox Sang 1979;36:50-64.

4 Statement from the consensus conference on anti-D prophylaxis. 7 and 8 April 1997. The Royal College of Physicians of Edinburgh. Royal College of Obstetricians and Gynaecologists. Vox Sang 1998:74:127-8.

5 Thornton JG, Page C, Foote G, et al. Efficacy and long term effects of antenatal prophylaxis with anti-D immunoglobueffects of antenatal prophylaxis
lin. BMf 1989;298:1671-3.

6 Hermann M, Kjellman H. Rh-prophylaxis with immunoglobulin anti-D administered during pregnancy and after delivery. Acta Obstet Gynecol Scand 1976;49(suppl):1-11. 